\title{
Treatment of nonocclusive mesenteric ischemia with type $B$ aortic dissection using intra-arterial catheterization after trauma surgery: case report
}

\author{
Sho Fujiwara ${ }^{1,2^{*}}$, Yuki Sekine ${ }^{2,3}$, Ryuichi Nishimura², Kazuya Tadasa ${ }^{2}$ and Shukichi Miyazaki ${ }^{2}$
}

\begin{abstract}
Background: Nonocclusive mesenteric ischemia (NOMI) is a mesenteric arterial spasm and intestinal ischemia. This disease is a highly lethal disease because diagnosis and decision of appropriate treatments are often difficult. Operations cannot resolve the spasms and may worsen the situation. However, the safety and effectiveness of catheterization for NOMI with aortic dissection (AD) have not yet been elucidated. Here, we report a successful case of early diagnosis and treatment of NOMI with type B AD involving the superior mesenteric artery (SMA) using the intra-arterial infusion of a vasodilator via the SMA.

Case presentation: An 83-year-old man was admitted to our hospital because of abdominal pain after a motor accident. We performed intestinal resection and splenectomy for intestinal perforation and splenic hemorrhage and treated conservatively for acute AD, liver injury, renal hematoma, and pneumothorax. On postoperative day (POD) 2, the patient had localized abdominal pain. Follow-up computed tomography suggested a smaller superior mesenteric vein sign and segmental lack of enhancement in the intestinal wall and ascites without SMA occlusion. Thus, the patient was diagnosed with NOMI. Although the patient had type B AD including the SMA, we performed selective mesenteric arteriography and transcatheter papaverine infusion via the SMA and prostaglandin via the peripheral vein. Seven days post treatment, mesenteric blood flow improved and intestinal wall enhancement was restored.
\end{abstract}

Conclusion: The intra-arterial infusion of a vasodilator is highly efficient and safety treatment option for NOMl with type B AD. Prompt and accurate management can prevent massive small bowel resection, and this procedure is essential in resolving a spasm independent of whether a necrotic bowel has been resected.

Keywords: Nonocclusive mesenteric ischemia, Type B aortic dissection, Intra-arterial catheterization, Spasm, Trauma

\section{Background}

Nonocclusive mesenteric ischemia (NOMI) refers to a mesenteric arterial spasm and intestinal ischemia and is a highly lethal disease; the mortality rate of NOMI is reported to be $70-90 \%[1,2]$. Treatments include the catheter-directed and infusion of vasodilatory and antispasmodic agents and open surgery, which is often required for necrotic bowels

\footnotetext{
* Correspondence: sho.fujiwara@med.tohoku.ac.jp

'Department of Molecular Pathology, Tohoku University School of Medicine, Sendai, Miyagi, Japan

2Department of Surgery, Iwate Prefectural Chubu Hospital, Kitakami, Iwate, Japan

Full list of author information is available at the end of the article
}

[3]. Open surgery cannot resolve the spasms and may worsen the situation [3]. However, the safety and effectiveness of catheterization for NOMI with type B aortic dissection (AD) have not yet been elucidated [1]. Here, we report the case of a patient with NOMI having type $\mathrm{B} A D$ involving the superior mesenteric artery (SMA) after a motor accident who was successfully treated using the intra-arterial infusion of a vasodilator via the SMA.

\section{Case presentation}

An 83-year-old man was admitted to our hospital because of abdominal pain after a motor accident. Focused 


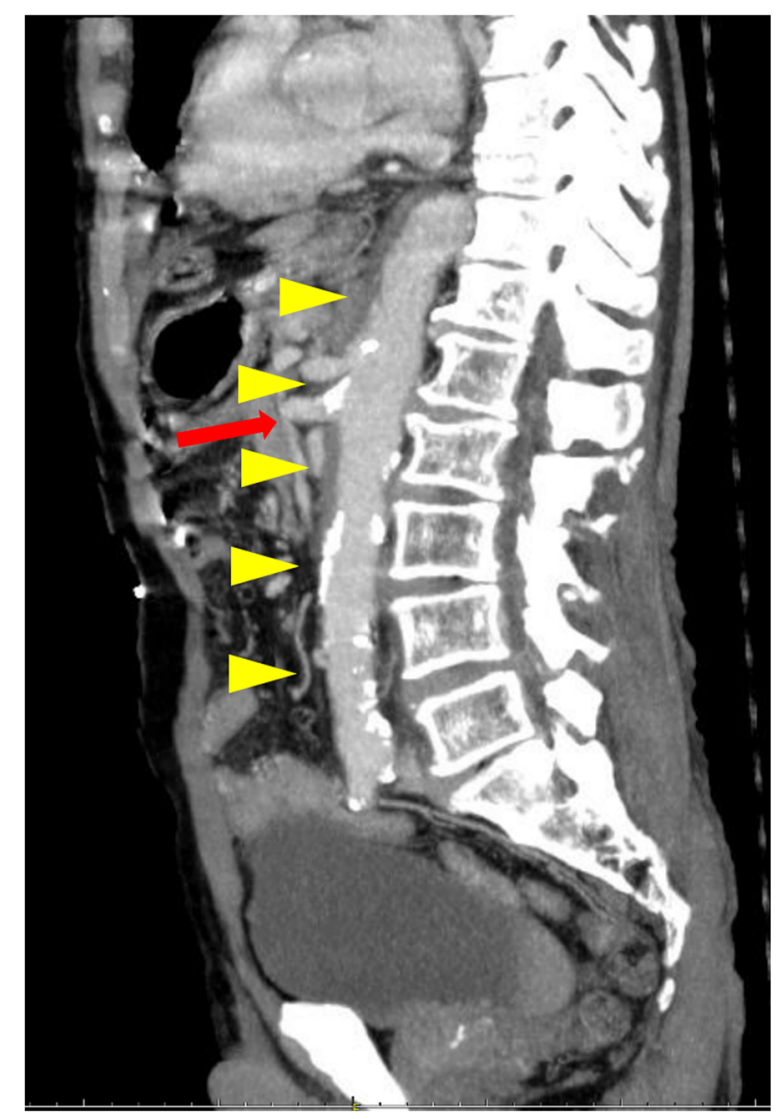

Fig. 1 Contrast-enhanced CT indicated a type B AD (yellow arrowheads) including the SMA (red arrow) assessment with sonography suggested the presence of blood in the abdomen. Contrast-enhanced computed tomography (CT) was performed (Fig. 1), which indicated intestinal perforation, acute $\mathrm{AD}$, liver injury, renal hematoma, and pneumothorax. Intestinal resection (the $10 \mathrm{~cm}$ of ileum was resected about $50 \mathrm{~cm}$ from the ileoceacal valve) and splenectomy were performed, and other injuries were conservatively treated. On postoperative day 2 , the patient experienced localized abdominal pain with defense and serum creatinine kinase (CK), lactate dehydrogenase (LDH), aspartate transaminase (AST), and lactate levels were elevated (Fig. 2). Then, D-dimer was $6.9 \mu$ g. Follow-up CT within an hour after the onset of symptoms revealed a smaller superior mesenteric vein sign and segmental lack of enhancement in the intestinal wall and ascites without SMA occlusion; the $\mathrm{CT}$ values of ischemic intestinal wall were approximately 5 to 25 Hounsfield unit (HU); ileum was 5 to 25 $\mathrm{HU}$ and jejunum was 65 to $90 \mathrm{HU}$ (Fig. 3a). Therefore, the patient was diagnosed with NOMI, localized peritonitis, and still reversible bowel ischemia. Although selective mesenteric arteriography and intra-arterial vasodilators were considered, the patient had type $\mathrm{B} A D$ including the SMA (Fig. 3b), and the insertion of a catheter was considered hazardous. Furthermore, he had acute respiratory distress syndrome, which made it difficult to perform laparotomy. We decided to perform selective mesenteric arteriography with the support of a vascular surgeon 5 hours after the onset of abdominal pain (Fig. 4a). The SMA was cannulated with an 150-cm long, 0.035 -in. thick, angle-type Radifocus ${ }^{\circ}$ guidewire (Terumo, Tokyo, Japan) and a 4-French Glidecath $\mathrm{II}^{\circ}$ shepherd-hook CJ2 (Terumo, Tokyo, Japan). The

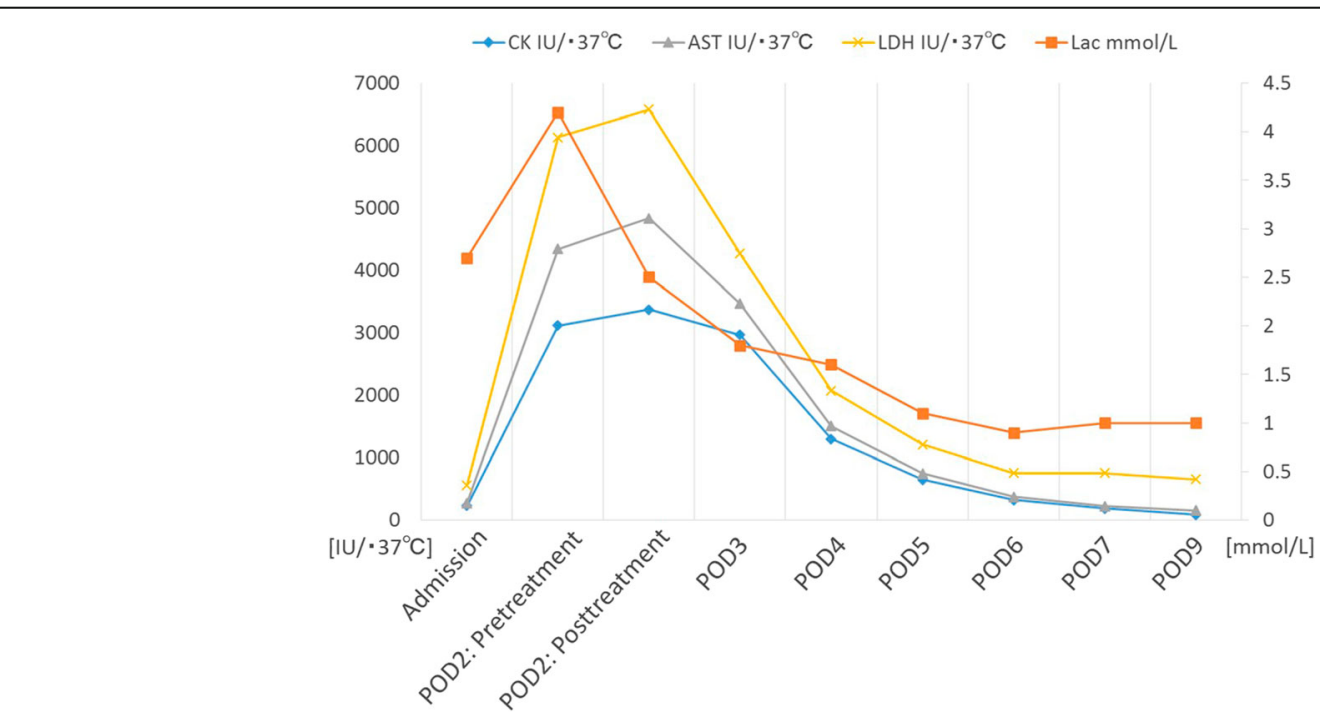

Fig. 2 Serum creatinine kinase (CK), lactate dehydrogenase (LDH), aspartate transaminase (AST), and lactate levels were elevated on POD 2. The intra-arterial infusion of a vasodilator improved laboratory data 


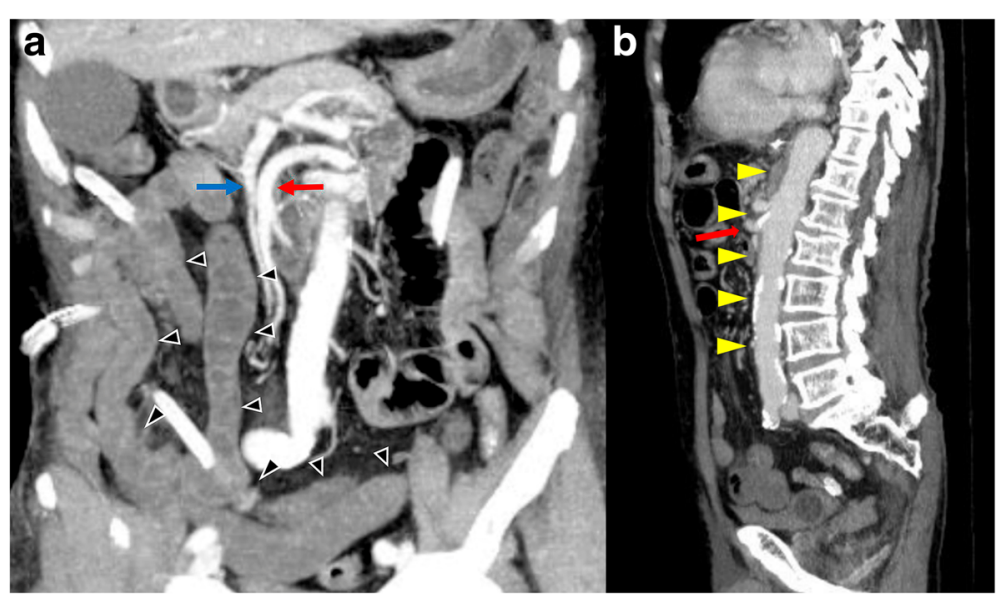

Fig. 3 a, b Follow-up CT indicated an AD (yellow arrowheads), smaller SMV sign, and segmental lack of enhancement in the intestinal wall (arrowheads). SMV (blue arrow) was smaller than SMA (red arrow)

infusion of papaverine $40 \mathrm{mg}$ and normal saline $19 \mathrm{~mL}$ after arteriography restored the blood flow (Fig. 4b). We then started transcatheter papaverine infusion $(28 \mathrm{mg} / \mathrm{h})$ via the SMA from 5-French Anthron ${ }^{\circ} \mathrm{P}-\mathrm{U}$ catheter $70 \mathrm{~cm}$ (Toray, Tokyo, Japan) and prostaglandin $(30 \mu \mathrm{g} /$ h) via the peripheral vein. Seven days post treatment, mesenteric blood flow improved (Fig. 5) and intestinal wall enhancement was restored. We did not perform any additional operation, and the patient was discharged from our hospital.

\section{Discussion}

We present a successful case of catheter-directed infusion into the SMA for a patient with NOMI complicated with traumatic type B AD involving the SMA. Vasodilator infusion via arterial catheter has been reported as an effective treatment for NOMI and many reports suggested efficacy of this strategies [4-7]. In 1977, Boley et al. decreased mortality rate from about $70-80 \%$ to $40 \%$ performing catheter intra-arterial infusion of papaverine
[8]. Other recent study revealed vasodilator administration from SMA achieved successful treatment in 64\% patients of NOMI after open heart surgery with nonsurgical treatment [7]. The strategy and management of NOMI with type $\mathrm{B} A D$ involving the SMA have not yet been established, and research is limited [1]. Thus, there are two overwhelming difficulties: risk from the catheterization of the dissected aorta and the accurate and prompt evaluation of NOMI.

First, the catheterization of and arterial infusion for the dissected aorta are essential, and more safety procedures were required than expected. We performed catheter-directed infusion of a vasodilator into the SMA because we believe that vasodilator therapies are the most important management option for all patients with NOMI; the resection of necrotic bowels cannot resolve spasms, which can recur by additional invasion, especially in such severe conditions [3]. Although treatments with catheter and cannulation into the SMA in the dissected aorta are essential techniques, this procedure

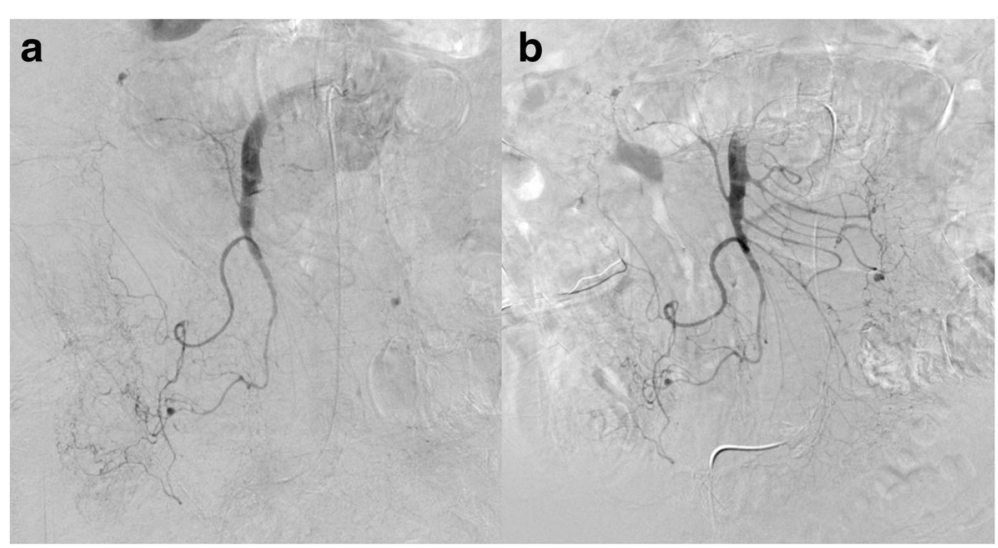

Fig. 4 a, b: Selective mesenteric arteriography (a). Infusion of papaverine restored the blood flow (b) 


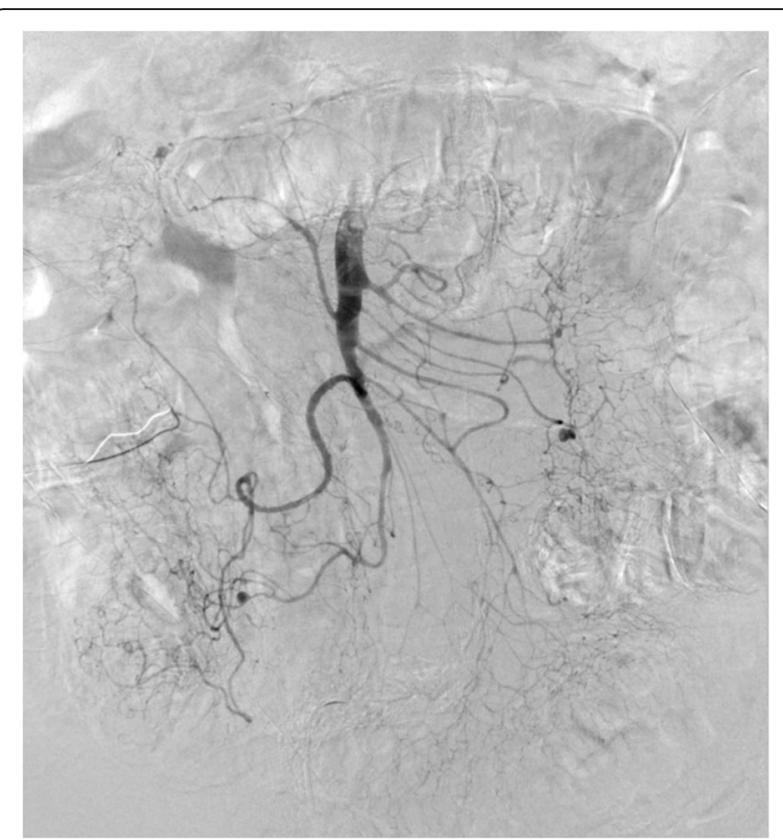

Fig. 5 Seven days posttreatment with transcatheter papaverine infusion from the SMA and prostaglandin from peripheral vein. Mesenteric blood flow was improved

is safe and highly efficient [3]. Recent developments in endovascular techniques and instruments enable us to perform more safety procedures than in the previous era; thoracic endovascular aortic repair and stenting for isolated SMA dissection are good examples [9]. We have to be careful in order to prevent an additional tear of the dissected aorta. However, these similar treatments have already been established as safety procedures, and cannulation of the dissected SMA has been performed during other endovascular treatments.

Second, contrast-enhanced CT considering vital signs, physical examinations, and laboratory data is essential for the accurate and prompt evaluation of NOMI [10]. CT imaging is a highly specific method for the diagnosis and evaluation of necrotic bowels [10, 11]. Although there have been reports that contrast-enhanced CT is not highly sensitive, the careful follow-up of laboratory data (lactate, CK, and LDH), abdominal pain, and vital signs can alert us regarding NOMI and bowel necrosis [11-13]. Furthermore, we apply this imaging for following of NOMI. We did not perform second-look laparotomy in this case because of the lack of intramural enhancement, and laboratory data improved after intra-arterial infusion therapy. However, if initial infusion of papaverine did not restore the spasm during angiography, we should keep in our mind and rule out other mesenteric ischemic diseases. In this case, our recommendations are arterial infusion therapy with careful observation considering laparoscopic exploratory laparotomy when bowel necrosis are suspected.

\section{Conclusions}

The intra-arterial infusion of a vasodilator is highly efficient and can be considered as a feasible treatment option for NOMI with type B AD. Prompt and accurate management can prevent massive small bowel resection. Furthermore, this procedure is essential in resolving a spasm independent of whether a necrotic bowel has been resected.

\section{Abbreviations \\ AD: Aortic dissection; AST: Aspartate transaminase; CK: Creatinine kinase; \\ CT: Computed tomography; HU: Hounsfield unit; LDH: Lactate \\ dehydrogenase; NOMI: Nonocclusive mesenteric ischemia; \\ POD: Postoperative day; SMA: Superior mesenteric artery}

\section{Acknowledgements}

We would like to appreciate the Enago for editing the English language.

Funding

None.

\section{Authors' contributions}

SF performed the operation, contributed to the acquisition of clinical data, and wrote the manuscript. YS, RN, KT, and SM performed the operation and contributed to the drafting and revision of the manuscript. All authors read and approved the final manuscript.

\section{Ethics approval and consent to participate}

Not applicable.

\section{Consent for publication \\ Informed consent was obtained from the patient and his family for this publication.}

\section{Competing interests}

The authors declare that they have no competing interests.

\section{Publisher's Note}

Springer Nature remains neutral with regard to jurisdictional claims in published maps and institutional affiliations.

\section{Author details}

${ }^{1}$ Department of Molecular Pathology, Tohoku University School of Medicine, Sendai, Miyagi, Japan. ${ }^{2}$ Department of Surgery, Iwate Prefectural Chubu Hospital, Kitakami, Iwate, Japan. ${ }^{3}$ Department of Vascular Surgery, Iwate Prefectural Chubu Hospital, Kitakami, Iwate, Japan.

Received: 23 October 2017 Accepted: 21 December 2017

Published online: 08 January 2018

\section{References}

1. Neri E, Sassi C, Massetti M, Roviello F. Nonocclusive intestinal ischemia in patients with acute aortic dissection. J Vasc Surg. 2001;36(4):738-46.

2. Park WM, Gloviczki P, Cherry KJ, Hallett JW, Bower TC, Panneton JM, et al. Contemporary management of acute mesenteric ischemia: factors associated with survival. J Vasc Surg. 2002;35:445-52.

3. Mitsuyoshi A, Tachibana T, Kondo Y, Momono T, Aoyama H. What we can learn from cases of synchronous acute mesenteric obstruction and nonocclusive mesenteric ischemia: how to reduce the acute mesenteric ischemia-related mortality rate. Ann Vasc Surg. 2016;32:133.

4. Habboushe F, Wallace HW, Nusbaum M, Baum S, Dratch P, Blakemore WS. Nonocclusive mesenteric vascular insufficiency. Ann Surg. 1974; 180(6):819-22.

5. Services TM, City B. Vasodilatory drugs in the management of nonocclusive bowel ischemia. Gastroenterology. 1975;68(1):146-50.

6. Clair DG, Beach JM. Mesenteric ischemia. N Engl J Med. 2016;374:959-68. 
7. Klotz S, Vestring T, Rötker J, Schmidt C, Scheld HH, Schmid C. Diagnosis and treatment of nonocclusive mesenteric ischemia after open heart surgery. Ann Thorac Surg. 2001;72(5):1583-6.

8. Boley S, Sprayregan S, Siegelman S, Veith F. Initial results from an aggressive roentgenological and surgical approach to acute mesenteric ischemia. Surgery. 1977:82(6):848-55

9. Luan JY, Li X, Li TR, Zhai GJ, Han JT. Vasodilator and endovascular therapy for isolated superior mesenteric artery dissection. J Vasc Surg. 2012;57(6): 1612-20.

10. Aschoff AJ, Stuber G, Becker BW, Hoffmann MHK, Schmitz BL, Schelzig $\mathrm{H}$, et al. Evaluation of acute mesenteric ischemia : accuracy of biphasic mesenteric multi-detector CT angiography. Abdom Imaging. 2009:34:345-57.

11. Mitsuyoshi A, Obama K, Shinkura N, Ito T, Zaima M. Survival in nonocclusive mesenteric ischemia. Ann Surg. 2007;246(2):229-35.

12. Chuong AM, Corno L, Beaussier H, Boulay-Coletta I, Millet I, Hodel J, et al. Assessment of bowel wall enhancement for the diagnosis of intestinal ischemia in patients with small bowel obstruction: value of adding unenhanced CT to contrast-enhanced CT. Radiology. 2016;280(1):98-107.

13. Furukawa A, Kanasaki S, Kono N, Wakamiya M, Tanaka T, Takahashi M, et al. $C T$ diagnosis of acute mesenteric ischemia from various causes. Am J Roentgenol. 2009:192:408-16.

\section{Submit your manuscript to a SpringerOpen ${ }^{\circ}$ journal and benefit from:}

- Convenient online submission

- Rigorous peer review

- Open access: articles freely available online

- High visibility within the field

- Retaining the copyright to your article

Submit your next manuscript at $\boldsymbol{s p r i n g e r o p e n . c o m ~}$ 